\title{
Flexible Estimation and Inference Within General Equilibrium Systems
}

\author{
Joseph Francois \\ Tinbergen Institute and CEPR
}

This version: June 2001 (revised)

\begin{abstract}
This paper explores estimation of parameters within large general equilibrium models, given limited data availability and without calibration. This leads directly to consideration of possible measures of the robustness of the model vis-à-vis the historical record (as represented by available data series), and the use of specification tests for comparison and testing of different generalequilibrium based theoretic paradigms. Such an approach also offers an opportunity for assessment of general equilibrium effects (like the impact of trade on wages or on the location of industry) currently highlighted in the policy literature.
\end{abstract}

Thanks are due to the participants in a CEPR/GIIS Geneva conference on empirical aspects of trade and location modeling, held at Villars Switzerland. Thanks are also due to Doug Nelson for helpful discussion. As usual, all errors are due to confusion on the part of the author. 


\section{INTRODUCTION}

Both econometrics and general equilibrium theory, along with their marriage in simultaneous equation modeling, share common roots in the work of the Cowles Commission for Research in Economics in the 1930s through 1950s. Yet, like many relationships, the one between theory and data has been somewhat stormy, particularly vis-à-vis empirical policy models. While the task set by the Cowles Commission at the time was for theory and data to work together (Koopmans 1953), today their partnership is best characterized as strained, perhaps even distant. Within macroeconomics, a large population of econometric modelers have largely given up on theory-based model specification, following on a research path of model specification guided primarily by data. ${ }^{1}$ Following the opposite approach, there is a large population of general equilibrium modelers who force the data to fit the dictates of theory. New generations of econometricians and general equilibrium modelers are being trained in one area with little emphasis on the other.

When we focus on trade and growth, this difficult relationship lurks behind differences between two empirical approaches to the quantitative analysis of economic structure and economic policy. One stream involves the application of econometric techniques to examine the historical record. In the area of international economics and growth, this includes, for example, the now extensive cross-country growth literature. It also includes the literature on globalization and labor markets, the literature on technology spillovers, and the literature on linkages between international capital markets and national performance. The second stream involves the use of calibration models. Small calibrated general equilibrium models are applied extensively in the real business cycle literature. Large calibrated general equilibrium models are used in the assessment of issues ranging from global trade liberalization to domestic tax reform and global-warming related emissions taxes. Justifiably, the growing use of calibrated models, without measures of confidence, and of goodness of fit (in short without measuring the models up against reality) has been accompanied by heavy criticism, especially from the econometrics community. Calibration modelers in turn criticize the more recent body of econometric exercises for sometimes

\footnotetext{
${ }^{1}$ However, there are ongoing efforts to again exploit theory within what have now become data-driven modeling exercises in macroeconomics. See the overview in Faust and Whiteman (1997).
} 
steering clear of or over simplifying important, but empirically complex policy issues. These are the household arguments that characterize the testy relationship between theory and data.

As they have evolved, there are important and obvious differences between econometric and calibration approaches to economic analysis. The econometric literature has emphasized testing, significance, and the robustness of results. It has also emphasized the narrowing down of problems to fit within the (frequently linear) econometric tools of choice. In contrast, the general equilibrium literature thrives on computational complexity, while often showing a blatant disregard for tracking actual data. Parameters are drawn from outside sources, while data are massaged (a two step process involving "construction of social accounting matrices" and also "calibration") to eliminate inconvenient incompatibilities between data and theory.

One branch of the general equilibrium ${ }^{2}$ literature well along the path to incorporating econometric techniques is the real business cycle literature. (See Kim and Pagan 1995). However, this literature deals with a much smaller, and analytically different, set of models than those used in the international trade, public finance, and development research communities. The latter models tend to be very large, multi-sector models with hundred or thousands of equations. In terms of testing with the larger models, emphasis has been on three issues: stochastic simulations for assessment of the distribution of parameters (see for example Arndt et al): econometric estimation of parameters (as in Jorgenson and Arndt et al); and model evaluation through ex post assessment (see Kehoe 1996 for an example). In general, the use of econometrics in this context has been driven by a need for parameters with which to feed the CGE models. Ex post assessments have been almost an afterthought, in reaction to critical questions by the policy community (a major consumer of these models) about how well the models actually perform.

This paper is concerned with estimation methods fitting within the confines of large general equilibrium models. This poses a number of problems. One is the fact that we immediately find ourselves working within a large system of non-linear simultaneous equations. Another is that the same parameters generally occur in more than one structural equation within the general equilibrium system, posing cross-restrictions. Most important,

\footnotetext{
${ }^{2}$ We should draw a distinction between the use of the term "general equilibrium" in macroeconomics and in trade. In macroeconomics, a model with one asset an one financial instrument may be called a general equilibrium model. In trade, these models can involve scores of sectors and regions and thousands of operational equations.
} 
the endogenous variables necessary to build a full time series are often unavailable, or worse still impossible to observe directly.

Different approaches have been taken to deal with these problems. The most common is just to give up on direct estimation of the structural parameters. Hence, a large share of the applied general equilibrium literature involves selection of parameters from the econometric ether, with remaining parameters being determined by theoretical relationships between these external parameters and base period data (i.e. calibration), as determined by the structural equations of the model. Of course, without direct estimation, we are at something of a loss if we need to assess the robustness of model results. For this reason, a literature has also emerged involving sensitivity analysis through stochastic simulations, where multiple simulations, with a ranging of parameter values based on prior distribution of the parameters, are used to assess the implied distribution of endogenous parameters. This approach provides some sense of how imposed parameters and their distributions affect the distribution of endogenous variables. In stark contrast to the econometric literature, however, no mapping is provided to actual data series. We are offered no measure of how the model actually tracks historical experience.

Because of concern about benchmarking to reality, there is also a small literature that takes large CGE models, and compares their results with historical experience. This includes, for example, Kehoe, who has examined a CGE model of Spain and the historical episode of accession to the European Community.

There have been notable efforts to estimate individual parameters within the context of a structural model. Foremost has been Dale Jorgenson, with various coauthors, who has employed non-linear econometric methods to estimate parameters within subsystems making up the production side of a general equilibrium model emphasizing energy use. More recently, Arndt and Robinson, with coauthors, have employed maximum entropy econometrics to estimate individual parameters within a system of general equilibrium equations. Their maximum entropy approach involves calibration to a base period. In both cases, the goal has ultimately been to develop parameter estimates for use within a calibrated model.

This paper addresses these issues collectively. In particular, it aims to explore systembased parameter estimation and specification testing, given limited data availability (and without calibration). This is linked to model robustness vis-à-vis the historical record (as represented by available data series). Given measures of CGE robustness, it also visits 
comparison and testing of alternative general equilibrium paradigms. Such an approach also offers opportunities for assessment of general equilibrium effects (like the impact of trade on wages or on the location of industry).

The structure of the paper is as follows. The next section lays out the structural model we work with. This model is a general equilibrium trade model that, while small, embodies the basic features of most CGE trade models. This is followed, in Section 3, by specification of the estimation problem. This is followed in Section 4 by a discussion of flexible estimation methods. Sections 5 and 6 then examine results from regressions with a synthetic dataset. Section 7 discusses confidence associated with model-based projections. Conclusions are offered in Section 8.

\section{EXPERIMENT DESIGN}

The approach in this paper involves working with a small general equilibrium trade model and a synthetic dataset. This allows us to compare estimated to "true" parameter values under alternative approaches to parameter estimation. The structural model is a stylized model of Armington-type trade along the lines developed by de Melo and Robinson (1989). Formally, we assume an economy where final demand is derived from a CES composite defined over a domestic good D and an imported good $\mathrm{M}$. The economy produces a domestic and export good E, with supply determined by a CET transformation technology between the two. Markets clear, and prices are competitive and inclusive of various tax wedges. A composite good Q serves all final demand channels. World prices are fixed (the small country assumption). The full model involves 19 equations and 19 exogenous variables, as summarized in equations (1) - (19) in Table 1. This specification follows Devarajan et al (1997). For the system in Table 1, our goal is to estimate the six relevant structural parameters: the CET transformation parameter $\Omega$; the CES substitution parameter $\sigma$; and weight and shift terms aq, at, bt, and bq.

The values of the parameters used for generation of the dataset are reported in Table 2. Based on the model detailed in Table 1, and the parameter values detailed in Table 2, a synthetic dataset has been generated for estimation purposes. The data generation process involves normal errors applied to the "real" endogenous variables resulting from the 
corresponding exogenous values. ${ }^{3}$ Given the model, and the dataset, in the next two sections we examine alternative methods for extraction of the model parameters.

Actual estimation is done in GAMS, using the CONOPT set of solvers. GAMS is a mathematical programming language well suited for large constrained maximization problems, like those described in the next sections. Conveniently, it is also the programming language of choice for large applied general equilibrium models. In the estimation process, the model is never actually "calibrated" at any point, though base period values are used to set initial variable levels within GAMS. Alternatively (and as discussed below) one can view the econometric estimation process as calibration to achieve a best fit to multiple data points.

While we know the true parameters, in cases where we do not the single equation regression estimates prove good initial guess values for the level of the structural parameters within the GAMS model.

\section{THE SYSTEM AND THE SUPER EQUATION}

We first examine parameter estimates given a complete dataset. In a sense, this serves as a benchmark relative to the flexible estimation approach discussed in the next section. To do this, we first rewrite the system in Table 1 more compactly.

First, consider our system as a set of implicit functions, such as market clearing identities, price index definitions, accounting identities, and the like. Our basic system as implicit functions can be expressed as follows:

$$
\boldsymbol{\varphi}=\left[\begin{array}{cccc}
\psi_{1}\left(\mathbf{Y}_{\mathbf{t}=1}, \mathbf{X}_{\mathbf{t}=1}: \boldsymbol{\theta}\right) & \psi_{2}\left(\mathbf{Y}_{\mathbf{t}=1}, \mathbf{X}_{\mathbf{t}=1}: \boldsymbol{\theta}\right) & \cdots & \psi_{g}\left(\mathbf{Y}_{\mathbf{t}=1}, \mathbf{X}_{\mathbf{t}=1}: \boldsymbol{\theta}\right) \\
\psi_{1}\left(\mathbf{Y}_{\mathbf{t}=2}, \mathbf{X}_{\mathbf{t}=2}: \boldsymbol{\theta}\right) & \psi_{2}\left(\mathbf{Y}_{\mathbf{t}=2}, \mathbf{X}_{\mathbf{t}=2}: \boldsymbol{\theta}\right) & \cdots & \psi_{g}\left(\mathbf{Y}_{\mathbf{t}=2}, \mathbf{X}_{\mathbf{t}=2}: \boldsymbol{\theta}\right) \\
\vdots & \vdots & \cdots & 0 \\
\psi_{1}\left(\mathbf{Y}_{\mathbf{t}=n}, \mathbf{X}_{\mathbf{t}=n}: \boldsymbol{\theta}\right) & \psi_{2}\left(\mathbf{Y}_{\mathbf{t}=n}, \mathbf{X}_{\mathbf{t}=n}: \boldsymbol{\theta}\right) & \cdots & \psi_{g}\left(\mathbf{Y}_{\mathbf{t}=n}, \mathbf{X}_{\mathbf{t}=n}: \boldsymbol{\theta}\right)
\end{array}\right]
$$

We can go further, and split the system up into two parts, corresponding to the left and right hand sides of the system of implicit functions (denoted as A and B where all equations are written as differences $\mathrm{A}-\mathrm{B}=0$ ). We then have:

\footnotetext{
${ }^{3}$ Exogenous variables are randomly adjusted as well from period to period, though they are assumed to be measured correctly.
} 


$$
\boldsymbol{\varphi}^{A}=\left[\begin{array}{cccc}
\psi_{1}^{A}\left(\mathbf{Y}_{\mathbf{t}=1}, \mathbf{X}_{\mathbf{t}=1}: \boldsymbol{\theta}\right) & \psi_{2}^{A}\left(\mathbf{Y}_{\mathbf{t}=1}, \mathbf{X}_{\mathbf{t}=1}: \boldsymbol{\theta}\right) & \cdots & \psi_{g}^{A}\left(\mathbf{Y}_{\mathbf{t}=1}, \mathbf{X}_{\mathbf{t}=1}: \boldsymbol{\theta}\right) \\
\psi_{1}^{A}\left(\mathbf{Y}_{\mathbf{t}=2}, \mathbf{X}_{\mathbf{t}=2}: \boldsymbol{\theta}\right) & \psi_{2}^{A}\left(\mathbf{Y}_{\mathbf{t}=2}, \mathbf{X}_{\mathbf{t}=2}: \boldsymbol{\theta}\right) & \cdots & \psi_{g}^{A}\left(\mathbf{Y}_{\mathbf{t}=2}, \mathbf{X}_{\mathbf{t}=2}: \boldsymbol{\theta}\right) \\
\vdots & \vdots & \cdots & 0 \\
\psi_{1}^{A}\left(\mathbf{Y}_{\mathbf{t}=n}, \mathbf{X}_{\mathbf{t}=n}: \boldsymbol{\theta}\right) & \psi_{2}^{A}\left(\mathbf{Y}_{\mathbf{t}=n}, \mathbf{X}_{\mathbf{t}=n}: \boldsymbol{\theta}\right) & \cdots & \psi_{g}^{A}\left(\mathbf{Y}_{\mathbf{t}=n}, \mathbf{X}_{\mathbf{t}=n}: \boldsymbol{\theta}\right)
\end{array}\right]
$$

$$
\boldsymbol{\varphi}^{B}=\left[\begin{array}{cccc}
\psi_{1}^{B}\left(\mathbf{Y}_{\mathbf{t}=1}, \mathbf{X}_{\mathbf{t}=1}: \boldsymbol{\theta}\right) & \psi_{2}^{B}\left(\mathbf{Y}_{\mathbf{t}=1}, \mathbf{X}_{\mathbf{t}=1}: \boldsymbol{\theta}\right) & \cdots & \psi_{g}^{B}\left(\mathbf{Y}_{\mathbf{t}=1}, \mathbf{X}_{\mathbf{t}=1}: \boldsymbol{\theta}\right) \\
\psi_{1}^{B}\left(\mathbf{Y}_{\mathbf{t}=2}, \mathbf{X}_{\mathbf{t}=2}: \boldsymbol{\theta}\right) & \psi_{2}^{B}\left(\mathbf{Y}_{\mathbf{t}=2}, \mathbf{X}_{\mathbf{t}=2}: \boldsymbol{\theta}\right) & \cdots & \psi_{g}^{B}\left(\mathbf{Y}_{\mathbf{t}=2}, \mathbf{X}_{\mathbf{t}=2}: \boldsymbol{\theta}\right) \\
\vdots & \vdots & \cdots & 0 \\
\psi_{1}^{B}\left(\mathbf{Y}_{\mathbf{t}=n}, \mathbf{X}_{\mathbf{t}=n}: \boldsymbol{\theta}\right) & \psi_{2}^{B}\left(\mathbf{Y}_{\mathbf{t}=n}, \mathbf{X}_{\mathbf{t}=n}: \boldsymbol{\theta}\right) & \cdots & \psi_{g}^{B}\left(\mathbf{Y}_{\mathbf{t}=n}, \mathbf{X}_{\mathbf{t}=n}: \boldsymbol{\theta}\right)
\end{array}\right]
$$

For estimation purposes, we employ a transformation of the system. In particular, we define the variables $\Phi_{\mathrm{it}}^{\mathrm{A}, \mathrm{B}}$ as elements of the vector of row sums of the matrix $\phi^{\mathrm{A}, \mathrm{B}}$, so that we have:

$$
\begin{aligned}
\boldsymbol{\Phi}_{t}^{A} & =\boldsymbol{\varphi}_{R, t}^{A} \\
\boldsymbol{\Phi}_{t}^{B} & =\boldsymbol{\varphi}_{R, t}^{B}
\end{aligned}
$$

We can then specify our transformed regression problem as follows:

$$
\hat{\mathbf{\Phi}}_{t}^{A}=\hat{\mathbf{\Phi}}_{t}^{B}\left(\hat{\mathbf{Y}}_{t}, \hat{\mathbf{X}}_{t}: \hat{\boldsymbol{\theta}}\right)+\hat{\boldsymbol{\gamma}}_{t}
$$

Note that we can apply weights to the elements of the $\phi^{\mathrm{A}, \mathrm{B}}$ matrix to put more or less weight on particular components of the system of equations (like market clearing in import markets, for example).

Instead of working with the indirect system of implicit functions as discussed above (and as presented in Table 1), we can also express the system as a direct one defined by implicit functions explicitly defining our endogenous variables. Splitting this direct system up into two parts, corresponding to the relevant left hand side (denoted $\mathbf{Y}$ ) and right hand side (denoted G), we have:

$$
\mathbf{Y}=\left[\begin{array}{cccc}
y_{1,1} & y_{2,1} & \cdots & y_{g, 1} \\
y_{1,2} & y_{2,2} & \cdots & y_{g, 2} \\
\vdots & \vdots & \cdots & \vdots \\
y_{1, n} & y_{2, n} & \cdots & y_{g, n}
\end{array}\right]
$$




$$
\mathbf{G}=\left[\begin{array}{cccc}
G_{1}\left(\mathbf{Y}_{\mathbf{t}=1}, \mathbf{X}_{\mathbf{t}=1}: \boldsymbol{\theta}\right) & G_{2}\left(\mathbf{Y}_{\mathbf{t}=1}, \mathbf{X}_{\mathrm{t}=1}: \boldsymbol{\theta}\right) & \cdots & G_{g}\left(\mathbf{Y}_{\mathbf{t}=1}, \mathbf{X}_{\mathbf{t}=1}: \boldsymbol{\theta}\right) \\
G_{1}\left(\mathbf{Y}_{\mathbf{t}=2}, \mathbf{X}_{\mathbf{t}=2}: \boldsymbol{\theta}\right) & G_{2}\left(\mathbf{Y}_{\mathbf{t}=2}, \mathbf{X}_{\mathbf{t}=2}: \boldsymbol{\theta}\right) & \cdots & G_{g}\left(\mathbf{Y}_{\mathbf{t}=2}, \mathbf{X}_{\mathbf{t}=2}: \boldsymbol{\theta}\right) \\
\vdots & \vdots & \cdots & 0 \\
G_{1}\left(\mathbf{Y}_{\mathbf{t}=n}, \mathbf{X}_{\mathbf{t}=n}: \boldsymbol{\theta}\right) & G_{2}\left(\mathbf{Y}_{\mathbf{t}=n}, \mathbf{X}_{\mathbf{t}=n}: \boldsymbol{\theta}\right) & \cdots & G_{g}\left(\mathbf{Y}_{\mathbf{t}=n}, \mathbf{X}_{\mathbf{t}=n}: \boldsymbol{\theta}\right)
\end{array}\right]
$$

For estimation purposes, we will again employ a transformation of the system. We again define the variables $\Phi_{\mathrm{it}}^{\mathrm{Y}}{ }^{\mathrm{G}}$ as elements of the vector of row sums of the matrix $\mathbf{Y , G}$.

$$
\begin{aligned}
\boldsymbol{\Phi}_{t}^{Y} & =\mathbf{Y}_{R, t} \\
\boldsymbol{\Phi}_{t}^{G} & =\mathbf{G}_{R, t}
\end{aligned}
$$

We can then specify our transformed regression problem as follows ${ }^{4}$ :

$$
\hat{\boldsymbol{\Phi}}_{t}^{Y}=\hat{\boldsymbol{\Phi}}_{t}^{G}\left(\hat{\mathbf{Y}}_{t}, \hat{\mathbf{X}}_{t}: \hat{\boldsymbol{\theta}}\right)+\hat{\mathbf{u}}_{t}
$$

For estimation purposes, we will employ the transformations of the system along the lines of equations (24) and (28). Our goal will be to estimate the parameter set $\theta$, using elements $\Phi$ of the set $\boldsymbol{\Phi}$ as the dependent variables. Obviously, for the indirect system we do not actually observe elements $\Phi$, and will instead use model-based estimates of these values to produce synthetic error term estimates. For the direct system, we do have (at least some) direct observations of $\mathbf{Y}$, though we will again employ the model itself to estimate missing observations.

Equations (24) and (28) summarize the stochastic system as a set of transformed variables. As we can employ any arbitrary weighting scheme to this transformation, there is a potentially infinite number of such transforms. We will refer to equations (24) and (26) as the super equation defining the system, and will focus on parameter estimation and specification testing in the context of these transformed system variables as represented by the super equation.

\footnotetext{
${ }^{4}$ Note that for equation (28), we can characterize the error term as $E(u)=\sum_{g} E(\varepsilon)$ and the variance of $u$ as $\sigma_{u}^{2}=\sum_{g} \sigma_{g}^{2}+\sum_{g} \sum_{j \neq g} \operatorname{cov}\left(\varepsilon_{g} \varepsilon_{j}\right)$, where $\boldsymbol{\varepsilon}$ denotes the error terms associated with $\mathbf{Y}$. In estimation, we then have the vector $\hat{\mathbf{u}}^{2}=\hat{\boldsymbol{\varepsilon}}^{\mathbf{T}} \hat{\boldsymbol{\varepsilon}}$. A similar identity holds for equation (24).
} 


\section{FleXiBLe Estimation}

We can view the parameter estimation problem as a special case of model selection. In particular, based on some model fitness criteria M, we are interested in the model, characterized by the parameter set $\hat{\boldsymbol{\theta}}$, that best meets M. (See the discussions in Davidson and MacKinnon 1993, Chapter 17; and in Bubna and Stewart 1998). The set of candidate models is bounded by our functional specifications and any bounds placed on $\boldsymbol{\theta}$. Obviously, there are different paths we can take at this point. This includes information-based model selection (like maximum entropy econometrics), Chi-squared selection rules (including maximum likelihood and generalized least squares regressions), or extensions involving bootstrap methods and synthetic datasets. (See the comparative discussion in Bubna and Stewart 1998 on the application of these methods to computer imaging). If we make the relatively weak assumptions that errors are zero mean and independent, then least squares estimates (with the zero mean condition) give us unbiased least variance estimates. (See Mardia, Kent, and Bibby 1979).

Whatever our starting point, we are likely to run into a problem common in general equilibrium modeling. Large theoretical general equilibrium models can describe tremendous amounts of data, by econometric standards. Assuming our methods imply a full-rank dataset, can we also hope to recover the underlying structural parameters of the model, given that real life is unfair? We will never really have large samples, and we will never have a full-rank set of endogenous variables.

To address this problem, we first turn back to our interpretation of equations (24) and (28). It helps to recall that we are not working with independent operations, but rather a system of operations that determines how the variables in the model interact. In this sense, any set of observations on a given endogenous variable provides information on the operation of the system as a whole. In this sense as well, the endogenous variables are signals sent by the system. We do not directly observe the working of the system (as represented by implicit functions). We do however observe how the signals interact. Combined with the structure of the model itself, these interactions provide information as to how the system as a whole operates. In this sense, even when we do not observe all endogenous variables, all variables we do observe give us additional information about the system as a whole (and hence implicitly about how well our functional representation of the system captures actual variable interactions). 
There are a number of reasons why general equilibrium modelers are unable to observe all endogenous parameters, even if the model is, by some odd chance, well specified vis-à-vis reality. One is that the models they work with involve signals and values, like CESutility based price indexes and utility, that simply will never be able to observe directly. Another is that, especially in a developing country context, data coverage may be spotty for institutional reasons.

So, if we move from large samples and full data series to smaller samples and more limited data series, how do we proceed? The next section of this paper examines smallsample properties, based on estimation of equations (24) and (28), for our working model. It also examines the use of flexible estimation methods, where we drop missing observations. To maintain the structure of the system, we replace missing values with estimated values, based on their implicit values as defined by the full system of equations. While we drop full series in the application, a similar approach can also be used to accommodate sporadic data series as well, filling sporadic holes in the data. As long as we approach the problem as one of estimating a collective system, as in equations (24) or (28), we have some flexibility in terms of data requirements, before the numeric problem degenerates excessively. In the present model, parameter estimates are relatively well behaved, even when some data series are available.

\section{Estimates}

We next turn to estimates. The basic estimation results are summarized in Table 3 and Figures 1 and 2. All estimates reported in Table 3 are based on repeated re-sampling of a sample population of 25 observations. As the name suggests, the full rank estimates involve a full rank dataset, with all endogenous observations present and accounted for. Estimation involves least squares, combined with the zero mean condition for the error terms $\mathbf{u}$ and $\boldsymbol{\gamma}$, applied to equation (24) - the indirect estimates, and to equation (28) - the direct estimates. Both are relatively robust, in that they are insensitive to starting points well away from final values. Standard errors are based on repeated random draws of the population of 25 (with replacement). ${ }^{5}$

\footnotetext{
${ }^{5}$ An alternative is to calculate error terms based on the matrix of partial derivatives in equation (24) and (28) with respect to the parameter values. Numeric point values can then be used to approximate the covariance
} 
While somewhat off, the parameter estimates are within the ballpark of actual values. In fact, based on the direct estimates, we can state that the correct or true values are within one standard deviation of the estimates. The same cannot be said for all the indirect estimates, as the estimate for OMEGA in particular appears to be biased in the small sample, in that we know the true value to be well outside the range suggested by our standard error estimate.

To shed some light on the small sample properties and the asymptotic properties of the estimators, Figures 1 and 2 present estimates with variations in sample size, based on random sample draws with replacement. As can be seen from the figures, the indirect estimates for both the substitution and transformation elasticity tend to wander in the parametric wilderness before converging, as the sample gets larger, on the true parameter values. They do eventually converge, though only as the sample gets very large. (In the context of time series data, we would probably need annual data series originating before the birth of Christ and ending with the second Millennium before we could feel confident of convergence to actual values). In contrast, the direct estimates converge rather rapidly on correct values. In this sense, one can be confident of being in the right parametric neighborhood with only a few decades of data. ${ }^{6}$

While not summarized in the tables and figures, a basic property of the estimators (at least in the context of our working model) seems to be that the indirect estimators are relatively robust in small samples, with rapid convergence to solution values. The indirect estimates are noisier, but are on average closer to actual values. There is an apparent tradeoff between the feasibility and robustness of the problem, and its precision vis-à-vis true values in a small sample setting.

Consider next the partial rank estimates in Table 3. Here we have dropped our observations of the Armington composite good quantities and prices, as well as internal import and export good prices: $\mathrm{P}_{\mathrm{q}}, \mathrm{Q}_{\mathrm{s}}, \mathrm{Q}_{\mathrm{d}}, \mathrm{P}_{\mathrm{e}}, \mathrm{P}_{\mathrm{m}}$. These values have instead been set equal to the functional estimates generated by the model the model: $\hat{P}_{q}, \hat{Q}_{s}, \hat{Q}_{d}, \hat{P}_{e}, \hat{P}_{m}$. In the context

matrix. In particular, we will have: $\hat{\sigma}_{\theta} \cong \sqrt{\frac{\sum \hat{u}^{2} /(n-1)}{\sum\left[\frac{\partial \hat{\phi}}{\partial \hat{\theta}}\right]^{2}}}$ See Davidson and MacKinnon (1993: Chapters 3and 8 ). 
of the direct estimates, this means we drop a set of error terms and endogenize the variable values. Identically, we produce synthetic observations with zero error terms. Note that there is virtually no change in parameter estimates, though there is an increase in the underlying degree of uncertainty. ${ }^{7}$

\section{Model Specification Tests}

We turn next to testing model specification. Given that we know the true model, it is easy to set up a theoretical straw man and knock him down. In this section, we have specified an alternative model, a nested version of the true model, wherein we assume that the CET surface is linear. This implies an infinite transformation elascticity, or identically it can be represented through modification to equations (1) and (4) in Table 1. For this example we work with the partial rank indirect estimates reported in Table 3.

The results of this exercise are summarized in Figures 3 and 4. The first reports the result (from 1,000 simulations) for a test statistic based on the Aikake Information Criterion (1973). Basically, assume we are considering two models (recall our discussion of parameter estimation as itself being a special case of model selection). Based on the Kullback-Leibler Information Criterion for measuring the adequacy of a model, we can compare our (nested) models on the basis of the following statistic:

$$
\begin{aligned}
& A I C=-2 \log L(\hat{\boldsymbol{\theta}})+2 k \\
& A I C_{1}-A I C_{2} \cong n \ln \left[\frac{\hat{\sigma}_{u, 1}^{2}}{\hat{\sigma}_{u, 2}^{2}}\right]-2\left(k_{1}-k_{2}\right)
\end{aligned}
$$

In equation (29), the estimated variance term $\hat{\sigma}_{u}^{2}$ is the estimated variance for the super equation. The rule is simply that if $A I C_{1}-A I C_{2}<1$, we choose model 1 (the general version), and when the value is greater than one, we choose the restricted model. Figure 3 presents the distribution of the AIC test statistic. Note that under this approach, we would reject the incorrect model roughly 95 percent of the time, based on our approximation to a distribution of 25 member samples.

\footnotetext{
${ }^{6}$ A question not yet dealt with is whether in a large sample "bootstrapped" from a small one, the parameter estimates converge on true values.

${ }^{7}$ For the present problem, relatively close parameter values (meaning in the neighborhood of 0.4 to 0.9 in the case of the CET and CES elasticities) can be recovered when only 3 or 4 variables are observed within the system.
} 
We can relate the AIC to the asymptotic $X^{2}$ tests. In particular, we can manipulate equation (29) to obtain an expression that is the function of sums of squares (and is hence characterized by an $\mathrm{F}$ distribution).

$$
F_{A I C} \cong \frac{\sigma_{1}^{2}-\sigma_{2}^{2}}{\sigma_{2}^{2}} \frac{n-k_{1}}{k_{1}-k_{2}} \sim F_{\left(k_{1}-k_{2}, n-k_{1}\right)}
$$

The implied critical value for this statistic, expressed in this way, will be

$$
F^{*}=\frac{n-k_{1}}{k_{1}-k_{2}}\left[e^{\frac{2\left(k_{1}-k_{2}\right)}{n}}-1\right]
$$

Which allows one to check the critical level of test from inverse F values from equation (31), with $\left(k_{1}-k_{2}\right)$ and $\left(n-k_{1}\right)$ degrees of freedom. In the present setting, this implies a threshold of .21 , instead of the .05 often assumed with classical $X^{2}$ tests.

To contrast the AIC-based test with a more classical test, Figure 4 presents Wald test statistics for the same experiments summarized in Figure 3. While the distributiuon implies that we are well within the bounds suggested by the AIC criteria (based on the critical F-level suggested by the AIC of 1.58 , or .22 certainty), we would fail to reject the wrong model at the .05 level roughly one-third of the time (based on a critical value of 4.38).

\section{Error Terms Associated With Model Projections}

There is a further issue to be addressed. This is the expression of confidence intervals surrounding projected variable values. In the CGE literature, emphasis has been on stochastic simulations for assessment of the impact of the parameter uncertainty (see for example Arndt).

We have several options here. In the context of direct parameter estimation, we can obviously use components of the matrix $\hat{\boldsymbol{\varepsilon}}$ for variables for which we had observations to begin with. For these variables, stochastic simulations are not necessary. For missing variables (those endogenized to obtain flexible estimates) we can still resort to stochastic simulation to approximate the relevant error terms. A similar approach can be followed, again along the lines of Arndt, when indirect estimation has been employed. 


\section{Conclusions}

Through work with a synthetic dataset, this paper highlights possibilities for estimation and inference within large structural and non-linear general equilibrium systems. Emphasis is placed on working with limited or incomplete datasets. The approach involves model transformation, combined with flexible estimation of the transformed system. This allows us to accommodate situations where not all series are directly observed, or where we have holes in out available time series. In the context of CGE models, unobservable values include variety-based price indexes and cost effects. While not directly observable, such variables are a critical part of the story. As shown here, we can construct, estimate, and work with such models even when critical endogenous variables remain unobserved. 


\section{References}

Akaike, H. (1973). "Information theory and an extension of the maximum likelihood princple." In B.N. Petrov and F. Csaki, editors, $2^{\text {nd }}$ International Symposium of Information Theory, Akademiai Kiado: 267-281

Arndt, C. (1996) "An Introduction to Systematic Sensitivity Analysis via Gaussian Quadrature.” GTAP Technical Paper No. 2, Center for Global Trade Analysis, Purdue University.

Arndt, C., S. Robinson, and F. Tarp. (1998). "Parameter estimation for a computable general equilibrium model: a maximum entropy approach.” Unpublished manuscript, IFPRI.

Bubna, K. and C.V. Stewart (1997). "Model Selection and Surface Merging in Reconstruction Algorithms." mimeo, Department of Computer Science, Rensselaer Polytechnic Institute. (a shorter version of this paper has appeared in Proc. IEEE Int. Conf. on Computer Vision, pp. 895-902, 1998).

Davidson, R. and J.G. MacKinnon (1993). Estimation and Inference in Econometrics. Oxford: Oxford University Press.

Devarajan, S., D.S. Go, J.D. Lewis, S. Robinson, and P. Sinko. (1998). Simple general equilibrium modeling." In J.F. Francois and K.A. Reinert, eds., Applied Methods for Trade Policy Analysis: A Handbook, Cambridge University Press: Cambridge UK.

Faust, J. and C.H. Whiteman (1997). "General to Specific Procedures for Fitting a DataAdmissible, Theory-Inspired, Congruent, Parsimonious, Encompassing, WeaklyExogenous, Identified, Structural Model to the DGP: A Translation and Critique." Board of Governors of the Federal Reserve System, International Finance Discussion Papers, Number 576 (March).

Gregory, A.W. and G.W. Smith (1993). "Statistical Aspects of Calibration in Macroeconomics." in G.S. Maddala, C.R. Rao, and H.D. Vinod eds., Handbook of Statistics, vol. 11, Elsevier Science Publishers.

Jorgenson, D.W. (1998). Growth: volume 1 - Econometric General Equilibrium Modelling. MIT Press: Cambridge MA.

T.J. Kehoe. (1996) "Social Accounting Matrices and Applied General Equilibrium Models." Federal Reserve Bank of Minneapolis, Working Paper Number 563.

Kim, K. and A.R. Pagan (1995). "The Econometric Analysis of Calibrated Macroeconmic Models." Handbook of Applied Econometrics, Basil-Blackwell. 
Koopmans, T.C. (1953). "Identification problems in economic model construction." Chapter 2 in W.C. Hood and T.C. Koopmans, eds., Studies in Econometric Method. New York: Wiley.

Liu, L., C. Arndt, and T. Hertel. (2000). "Calibration and validation of trade substitution parameters in GTAP model: a maximum entropy approach.” GTAP consortium.

Mardia, K.V., J.T. Kent, and J.M Biddy (1979). Multivariate Analysis. Academic Press.

Mariano, R.S. and B.W. Brown (1993). "Stochastic simulations for inference in nonlinear errors-in-variables models." in G.S. Maddala, C.R. Rao, and H.D. Vinod eds., Handbook of Statistics, vol. 11, Elsevier Science Publishers.

de Melo, J. and S. Robinson (1989). "Product differentiation and the treatment of foreign trade in computable general equilibrium models." Journal of International Economics 27:47-67.

Newey, W.K. (1993). "Efficient estimation of models with conditional moment restrictions." in G.S. Maddala, C.R. Rao, and H.D. Vinod eds., Handbook of Statistics, vol. 11, Elsevier Science Publishers. 
Table 1

(1) National Product Function: $X=a_{t} \cdot\left[b_{t} \cdot E^{r t}+\left(1-b_{t}\right) \cdot D^{r t}\right]^{1 / r t}$

(2) Armington Function: $\quad Q=a_{q} \cdot\left[b_{q} \cdot M^{r q}+\left(1-b_{q}\right) \cdot D^{r q}\right]^{1 / r q}$

(3) Aggregate Demand: $\quad Q^{D}=C+Z+\bar{G}$

(4) Relative Supply: $\quad E=D \cdot\left(\frac{P_{E}}{P_{D}}\right)^{\Omega} \cdot\left(\frac{b_{t}}{1-b_{t}}\right)^{\Omega}$

(5) Relative Demand: $\quad M=D \cdot\left(\frac{P_{D}}{P_{M}}\right)^{\sigma} \cdot T^{-\sigma} \cdot\left(\frac{b_{q}}{1-b_{q}}\right)^{-\sigma}$

(6) Tax Revenue:

$$
T=t_{M} \cdot P_{W M} \cdot E r \cdot M+t_{E} \cdot P_{E} \cdot E+t_{S} \cdot P_{Q} \cdot Q_{D}+t_{Y} \cdot Y
$$

(7) National Income: $\quad Y=P_{X} \cdot X+t_{R} \cdot P_{Q} \cdot Q+r e \cdot E r$

(8) Savings: $\quad S=s_{Y} \cdot Y+E r \cdot B+S_{G}$

(9) Consumption: $\quad C=Y \cdot\left(1-t_{Y}-s_{Y}\right)$

(10) Import Prices: $\quad P_{M}=E r \cdot P_{W M} \cdot\left(1+t_{M}\right)$

(11) Export Prices: $\quad P_{E}=E r \cdot P_{W E} /\left(1+t_{E}\right)$

(12) Consumer Prices: $\quad P_{T}=P_{Q} \cdot\left(1+t_{S}\right)$

(13) Output Prices: $\quad P_{X}=\frac{P_{E} \cdot E+P_{D} \cdot D_{S}}{X}$

(14) Supply Prices: $\quad P_{Q}=\frac{P_{M} \cdot M+P_{D} \cdot D_{D}}{Q_{S}}$

(15) Numeraire: $\quad E r=1$

(16) Domestic Market Clearing: $D_{D}=D_{S}$

(17) Composite Market Clearing: $Q_{D}=Q_{S}$

(18) Current Account Balance: $B=P_{W M} \cdot M-P_{W E} \cdot E-f t-r e$

(19) Government Budget: $\quad T=G \cdot P_{T}-t_{R} \cdot P_{Q}+f t \cdot E r$

Table 1 -- continued 


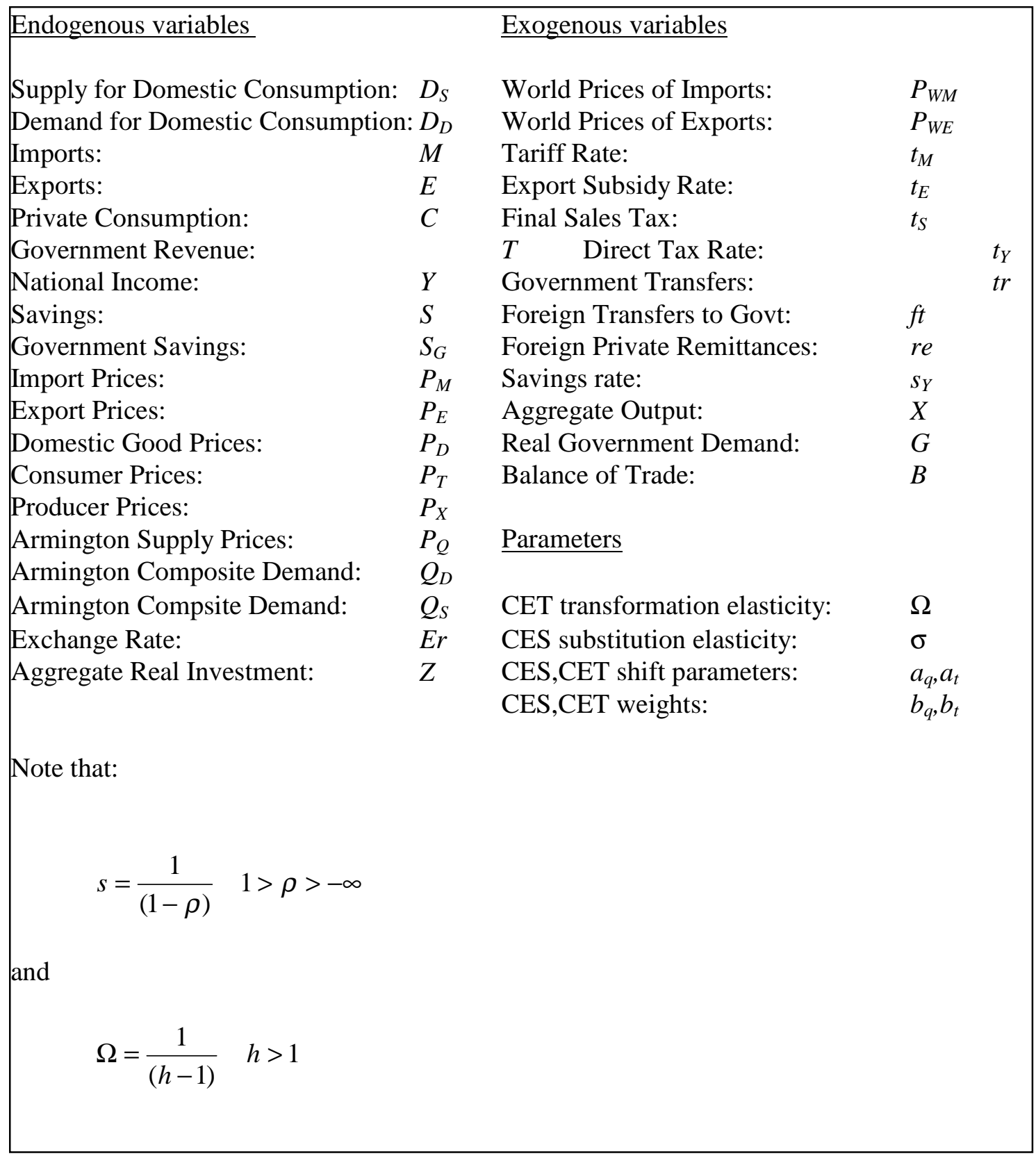


Table 2: $\quad$ Actual Parameter Values

\begin{tabular}{llll}
$\Omega:$ & 0.60 & $b_{q}:$ & 0.38 \\
$\sigma:$ & 0.60 & $a_{i}:$ & 2.22 \\
$b_{t}:$ & 0.77 & $a_{q}:$ & 1.97 \\
\hline
\end{tabular}


Table 3: $\quad$ Small-sample estimates of parameter values

FULL RANK SAMPLE

indirect estimates

\begin{tabular}{|c|c|c|c|}
\hline$\Omega:$ & $\begin{array}{l}0.70 \\
(.04)\end{array}$ & $b_{q}:$ & $\begin{array}{l}0.37 \\
(.02)\end{array}$ \\
\hline$\sigma:$ & $\begin{array}{l}0.58 \\
(.04)\end{array}$ & $a_{t}:$ & $\begin{array}{l}2.17 \\
(.05)\end{array}$ \\
\hline$b_{t}:$ & $\begin{array}{l}0.74 \\
(.01)\end{array}$ & $a_{q}:$ & $\begin{array}{l}1.96 \\
(.04)\end{array}$ \\
\hline \multicolumn{4}{|c|}{ direct estimates } \\
\hline$\Omega:$ & $\begin{array}{c}0.77 \\
(.17)\end{array}$ & $b_{q}:$ & $\begin{array}{l}0.37 \\
(.07)\end{array}$ \\
\hline$\sigma:$ & $\begin{array}{l}0.57 \\
(.10)\end{array}$ & $a_{t}:$ & $\begin{array}{l}2.17 \\
(0.4)\end{array}$ \\
\hline$b_{t}:$ & $\begin{array}{l}0.71 \\
(.13)\end{array}$ & $a_{q}:$ & $\begin{array}{l}1.97 \\
(.35)\end{array}$ \\
\hline
\end{tabular}

PARTIAL RANK SAMPLE

(observations for Qd, Qs, Pq, Pm, Pe are endogenized)

indirect estimates

\begin{tabular}{llll}
\hline$\Omega:$ & 0.59 & $b_{q}:$ & 0.36 \\
& $(.03)$ & & $(.02)$ \\
$\sigma:$ & 0.58 & & \\
& $(.04)$ & $a_{t}:$ & 2.20 \\
& & & $(.04)$ \\
$b_{t}:$ & 0.77 & $a_{q}:$ & 1.98 \\
& $(.01)$ & & $(.03)$
\end{tabular}

direct estimates

$\begin{array}{llll}\Omega: & 0.78 & b_{q}: & 0.36 \\ & (24) & & (.10) \\ \sigma: & 0.57 & & \\ & (.16) & a_{t}: & 2.17 \\ b_{t}: & 0.72 & & (.60) \\ & (.20) & a_{q}: & 1.97 \\ & & & (.54)\end{array}$

note: standard-errors, based on boot strap estimates, are reported in parentheses. 
Figure 1

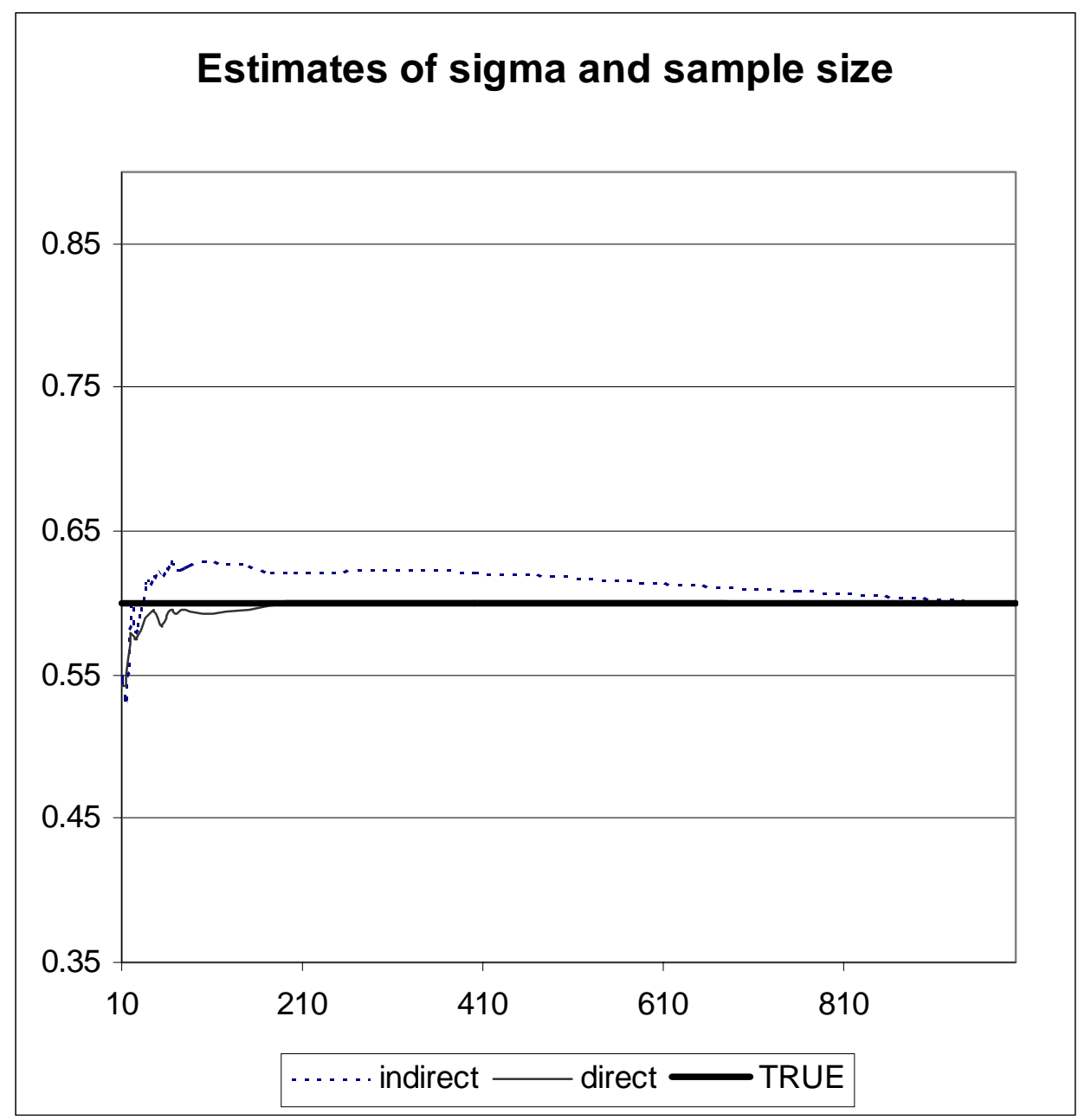

note: These involve flexible estimates, where Qd, Qs, Pq, Pe, and Pm are unobserved. 
Figure 2

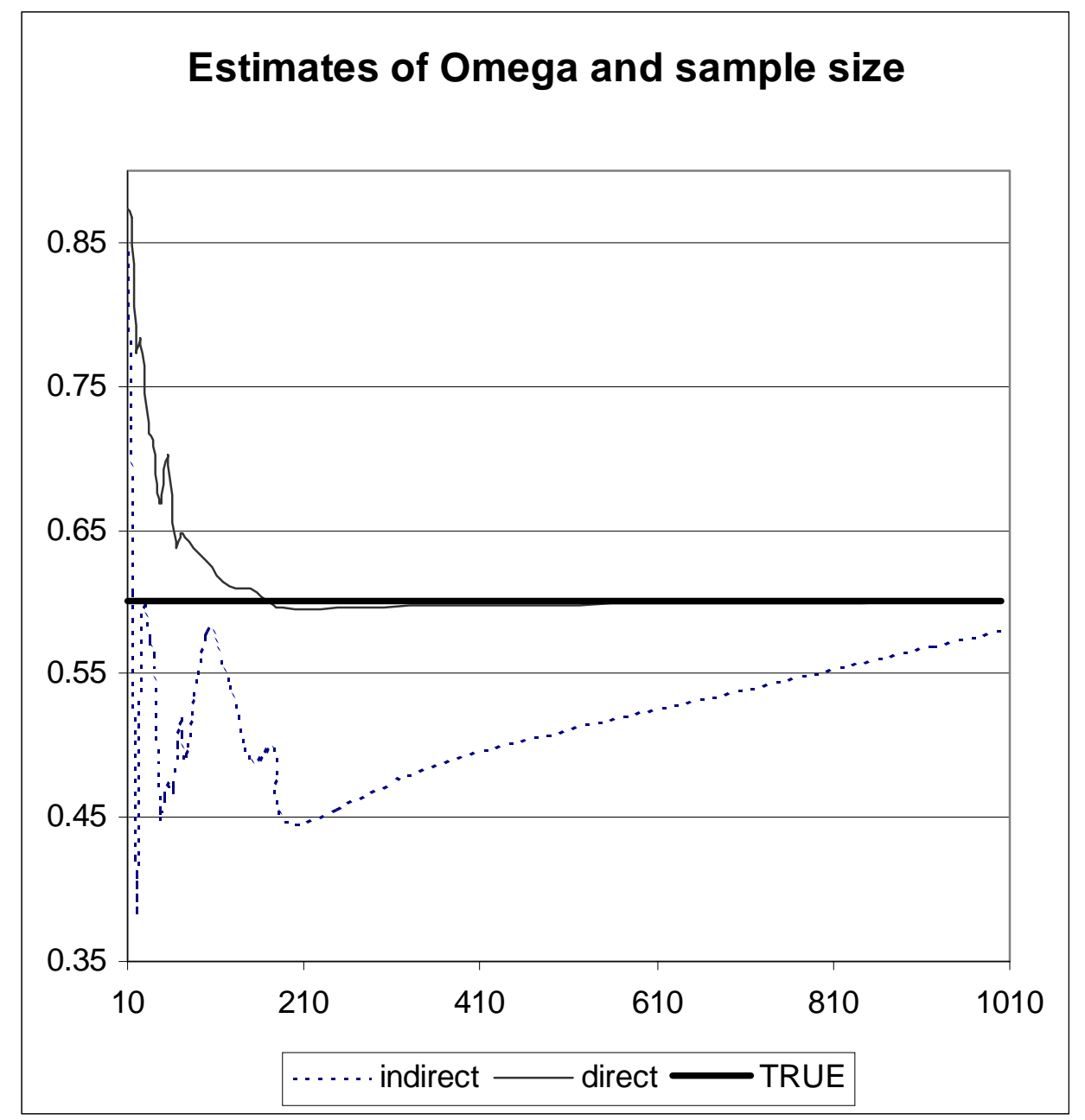

note: These involve flexible estimates, where $\mathrm{Qd}, \mathrm{Qs}, \mathrm{Pq}, \mathrm{Pe}$, and $\mathrm{Pm}$ are unobserved. 
Figure 3

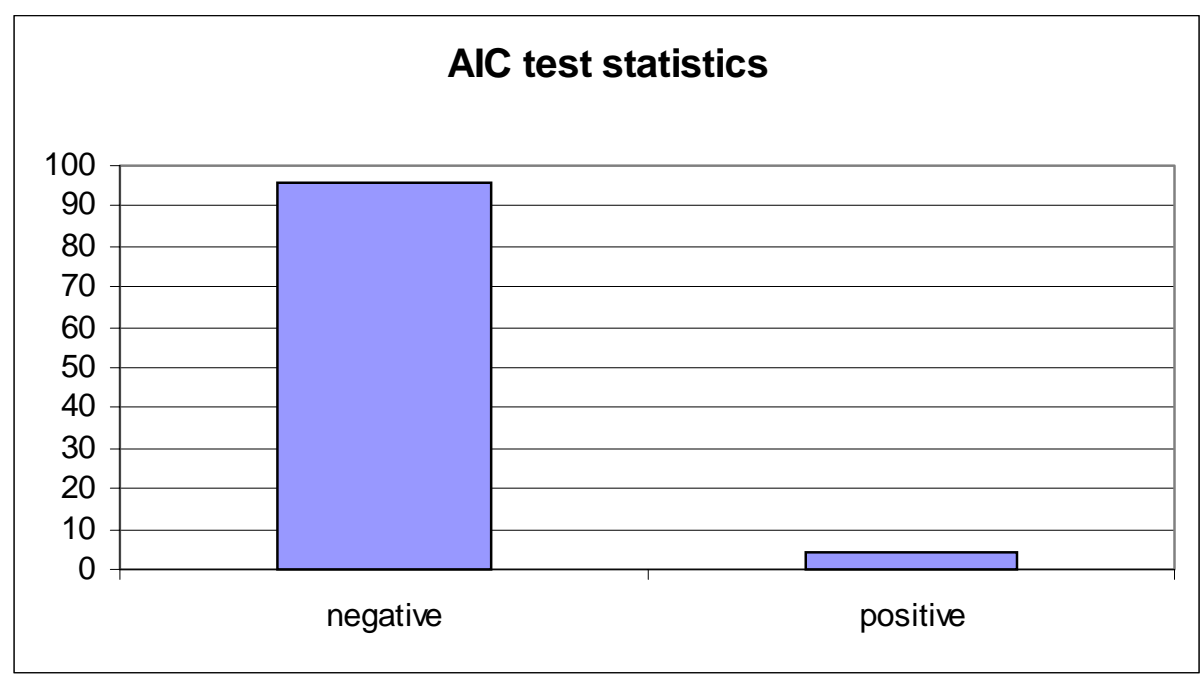

Figure 4

Pseudo-F Statistic

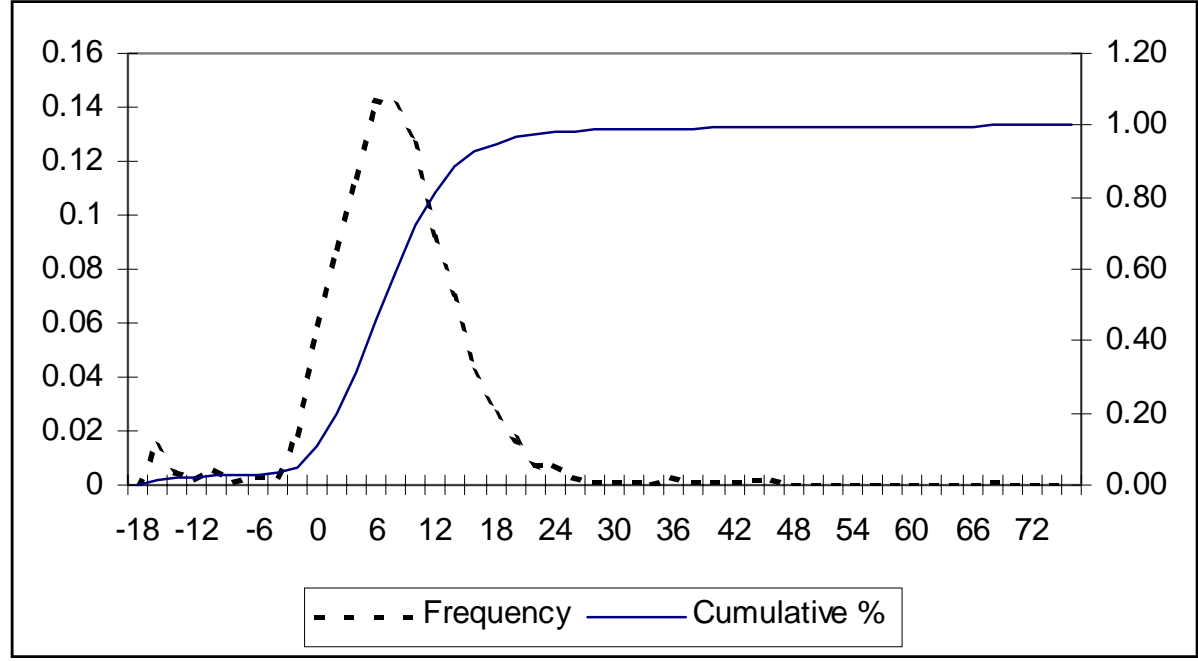

Note: these figures are for a the partial rank dataset and estimates detailed in Table 3.

The specification test involves a comparison of the model in Table 1 with a nested alternative model where the CET frontier is restricted to be linear. 